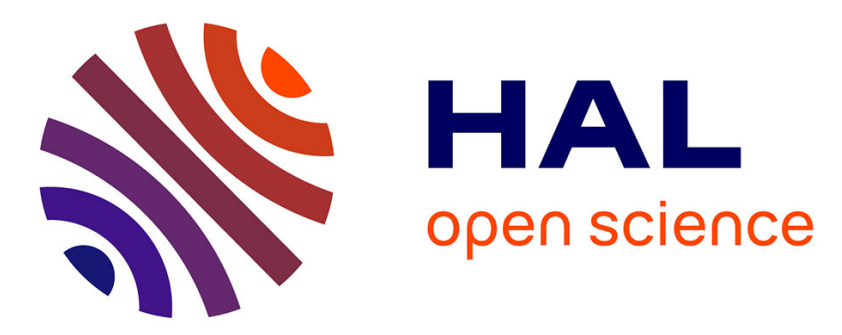

\title{
Lempel-Ziv complexity of the pNNx statistics -an application to neonatal stress
}

\author{
Matej Šapina, Chandan Karmakar, Karolina Kramarić, Marcin Kośmider, \\ Matthieu Garcin, Dario Brdarić, Krešimir Milas, John Yearwood
}

\section{To cite this version:}

Matej Šapina, Chandan Karmakar, Karolina Kramarić, Marcin Kośmider, Matthieu Garcin, et al.. Lempel-Ziv complexity of the pNNx statistics -an application to neonatal stress. 2019. hal-02163492

\section{HAL Id: hal-02163492 \\ https://hal.science/hal-02163492}

Preprint submitted on 24 Jun 2019

HAL is a multi-disciplinary open access archive for the deposit and dissemination of scientific research documents, whether they are published or not. The documents may come from teaching and research institutions in France or abroad, or from public or private research centers.
L'archive ouverte pluridisciplinaire HAL, est destinée au dépôt et à la diffusion de documents scientifiques de niveau recherche, publiés ou non, émanant des établissements d'enseignement et de recherche français ou étrangers, des laboratoires publics ou privés. 


\section{Lempel-Ziv complexity of the pNNx statistics - an application to neonatal stress}

Matej Šapina ${ }^{1,2,3}$, Chandan Karmakar ${ }^{4}$, Karolina Kramarić1, ${ }^{1,2,3}$, Marcin Kośmider ${ }^{5}$, Matthieu Garcin $^{6}$, Dario Brdarić3,7, Krešimir Milas ${ }^{1,2}$, John Yearwood ${ }^{4}$

${ }^{1}$ University hospital Osijek, Pediatric Clinic, J. Huttlera 4, 31000 Osijek, Croatia, ${ }^{2}$ Medical faculty Osijek, Osijek, J. Huttlera 4, 31000 Osijek, Croatia, ${ }^{3}$ Faculty of Dental medicine and Health, Crkvena 21, 31000 Osijek, Croatia, ${ }^{4}$ School of Information Technology, Deakin University, Geelong, Australia, ${ }^{5}$ Institute of Physics, University of Zielona Gora, Prof. Szafrana 4a, 65-516 Zielona Gora, Poland, ''éonard de Vinci Pôle Universitaire, Research center, 92916 Paris La Défense, France, ${ }^{7}$ Institute of Public Health for the Osijek Baranya County, Drinska 8, 31000 Osijek, Croatia

Corresponding author:

Matej Šapina, MD

J. Huttlera 4, 31000 Osijek, Croatia

Phone: 00385955567876

E-mail: sapina.matej@yahoo.com 


\section{ABSTRACT}

Among the existing measures of heart rate variability (HRV), the pNN50 statistics is one of the most commonly reported. However, it is only a single member of a much larger family of HRV measures - the pNNx statistics. In this research pNNx was further extended, combining it with the Lempel-Ziv complexity (LZ76) in a controlled neonatal stress framework. Forty healthy newborns were recorded undergoing two different types of stress stimuli - a routine heel stick blood sampling, and a dull heel pressure stimulation. Instead of relying on a single value, the entire spectrum from pNN1 to pNN100 was calculated, along with LZ76 derived from binarized sequences for each NN. The results of this study show a downward shift of the pNNx curves when newborns are stressed, with reduced LZ76 complexity when stressed. When ROC curves were utilized for the pNNx statistics and LZ76, however, the highest AUC values were observed when both measures were combined, with the highest AUC values of $0.88(0.80-0.94)$ and $0.85(0.74-0.91)$ for discriminating resting states from stress phases. Combining the widely used pNNx statistics with LZ76 extends the existing HRV toolbox, and shows a promising application in recognizing acute neonatal stress.

Keywords: Heart rate variability, Lempel-Ziv Complexity, Newborns, Stress, Cardiac inter-beat interval, Pain 


\section{INTRODUCTION}

Children differ from adults in various ways. Due to their dynamic developmental changes, the pediatric population is subjected to higher environmental polutants. The lower the newborn's gestational age, the less is their adaptive tolerance, leading to stress responses, which would not occur in the mature human ${ }^{1,2}$.

For a long period, a paradigm existed regarding the lack of capabilities of newborns for feeling pain. But when, in the late 1980s, Anand and Hickey published their seminal work on neonatal pain, the traditional views started to change ${ }^{3}$. However, newborn pain still remains undertreated ${ }^{4}$. Pain induces a stress response, which changes the bodies' homeostasis, and can have both a short- and long-term effect on the developing body ${ }^{5,6}$. When being stressed, a hormonal response is generated, leading to an increase of heart and respiratory rate, blood pressure fluctuations, changing the cerebral blood flow, and in the long run, even epigenetic changes might occur ${ }^{6-8}$.

Acute stress and newborn pain is commonly assesed using pain scales ${ }^{9}$. Unfortunately, relying only on pain scales might suffer from lack of objectivity, due to the subjective or incosistent pain assesment, and further due to the limited knowledge of the nature how neonates response to pain ${ }^{10}$. Both term and preterm newborns in the intensive care unit (NICU) are continuously being monitored using different devices, which can be used as an aid for a more objective stress and pain assessment ${ }^{11,12}$.

Heart rate variability (HRV) is the variability of duration of consecutive cardiac cycles originating from the sinus node. In the last decades, HRV showed itself useful as both a basic science reasearch tool for the assessment of the autonomic nervous system (ANS), as well as a prognostic tool in clinical applications ${ }^{13}$. HRV is assesed via different mathematical and 
statistical methods, which can be roughly divided into: time domain, frequency domain, and nonlinear methods ${ }^{14}$.

The time domain analysis can be further divided as measures directly and indirectly derived from the RR interval recording. Among the latter, probably the simplest to be calculated is the widely, and nowadays standardly used measure - the pNN50 statistic. The pNN50 statistics was initially developed by Ewing in 1984. It is defined as the proportion consecutive normal sinus (NN) intervals whose differences exceed $50 \mathrm{~ms}$. As such it was proposed to be a measure of parasympathetic activity ${ }^{15}$. Since then, the pNN50 proved itself to be a useful measure of HRV in various basic and clinical research. However, the fixed threshold of $50 \mathrm{~ms}$, was only arbitrarily recommended due to the easiness of calculation ${ }^{16}$.

Therefore, the pNN50 statistic is only a single member of a much larger family of HRV measures - the pNNx family. Mietus et al. first investigated the relationship of other thresholds compared to the standard pNN50, trying to find a more robust discrimination between healthy and diseased groups of patients ${ }^{16}$. As more evidence was gathered, lower $\mathrm{NNx}$ values are being more frequently reported along with pNN50.

Time and frequency domain are often not sufficient to describe the complex heart rate variability dynamics, due to the complex interactions of mechanisms involved in cardiovascular regulations ${ }^{17}$. Various nonlinear methods have been proposed in the analysis of HRV, among which complexity measures play a particular role ${ }^{18}$. The Lempel Ziv (LZ) complexity is a measure to assess the algorithmic complexity, defined according to the Information Theory as the least amount of information to describe a binary string ${ }^{19}$. With the time evolution of the extending string, the LZ complexity quantifies the rate of new patterns arising ${ }^{20,21}$. 
This research is based on the notion that even simple statistics such as the pNNx statistics can exhibit complex structures of the heart rate variability. We are particularly interested in applying the LZ complexity to a coarse-grained time series from the binarized pNNx statistics spectrum. Thus, we hypothesize the simultaneous application of pNNx and LZ will provide more useful discriminating properties than each statistic alone.

\section{SUBJECTS AND METHODS}

\section{Ethical statement}

The research was accepted by the University hospital Clinic Osijek ethical committee, and informed consents were obtained from all research participant's parents or guardians.

The study protocol

The patient's demographics and study protocol have been reported previously ${ }^{22-25}$. Shortly, forty healthy term neonates, born through vaginal delivery, with an APGAR score >9/9 were included in the study. The study sample consisted of 21 females and 19 males with birth weight $3542.05 \pm 339.09 \mathrm{~g}$, and were randomly chosen over a three months period from the Osijek University Clinic pediatrics clinic's maternity ward. Except two female twins, the rest of the participants were singletons. The study was performed when the newborns reached 72 hours postnatal age, just prior discharge, which is the recommended chronological age for routine phenylketonuria and congenital hypothesis screening. Besides the routine screening heel stick blood draw, none of the newborns underwent any other procedural pain.

The experimental protocol is divided into three parts: a) dummy stimulation, b) the sharp pain - heel stick, c) the treatment; however, the treatment part was not included in the study. Both part a) and b) consisted of two phases: a baseline resting phase (Phases 1 and 3), 
and an interventional phase (Phases 2 and 4). The protocol was carried out in a serialized manner: Phase 1) - 10 minutes of resting, followed by Phase 2) the dummy stimulation phase, where intermittent pressure onto the newborn's heel was applied, mimicking a heel stick blood drawing without blood sampling. The duration of Phase 2 was fixed at 90 seconds, which was the average time for the nurse to perform the blood drawing. At the end of Phase 2, Part b) of the experiment began. Phase 3 was the second resting phase, lasting 10 minutes, followed by the heel stick blood drawing procedure (Phase 4). Phase 4 differed in duration, because of the variability of time needed to collect the least amount of blood for the metabolic screening.

During the entire procedure, the RR intervals were collected with a light weight highresolution sampling device (Firstbeat Bodyguard 2, Firstbeat TechnologiesLtd, Jyvaskyla, Finland, sampling frequency $1024 \mathrm{~Hz}$ ). The recordings were further visually inspected, and after artifact removal, the data was ready for analysis. Each infant was fed with breast milk or formula, and positioned in supine position in a quiet room to minimize the external artefacts.

The $p N N x$ statistics and the Lempel-Ziv complexity

We simply calculated the pNNx statistics as the number of consecutive RR (NN) intervals differing by more than $x \mathrm{~ms}$ in the entire recording divided by the total number of all RR intervals (nNN):

$$
p N N x=\frac{\operatorname{Card}\left(\mathrm{i} \leq n N N,\left|\mathrm{RR}_{i+1}-\mathrm{RR}_{i}\right|>\mathrm{x}\right)}{n N N} .
$$




\section{Binarization}

A binarized sequence is the starting point to obtain both the pNNx statistics and LZ complexity. From the original RR interval time series, differences between two successive RR intervals greater than a threshold value $x$ are replaced with 1, and values lesser or equal to $x$, with 0 . Therefore, for each threshold $x$, both the pNNx and LZ complexity can be calculated:

1. The preprocessing step consists in calculating the first absolute differences between successive RR intervals. For example, the first absolute differences of the time series

$$
R R \equiv(343,350,349,400,551) \text { equal } \Delta R R \equiv(7,1,51,151)
$$

To find the pNN50, the number of elements greater than 50 is divided by the total number of elements, which in this example is pNN50 $=0.5$. Additionally, to calculate the pNN2, the numbers of elements greater than 2 is divided by the total numbers of elements in the time series, which is here pNN2 $=0.75$.

2. After defining the threshold number $x$, a sequence can be binarized by replacing the elements greater than $x$ in the $\Delta R R$ time series with 1, and the others with 0 .

If $x=50$, the first absolute differences time series, $\Delta R R \equiv(7,1,51,151)$, is converted into:

$$
\Delta \operatorname{RR}_{01}(50) \equiv(0,0,1,1)
$$

Additionally, if $x=2$, the time series becomes:

$$
\Delta R_{01}(2) \equiv(1,0,1,1) .
$$

3. After obtaining the binary sequence, the standard and normalized LZ76 can be calculated, as follows. 
The $L Z$ is often used as a parameter to estimate the complexity of discrete time series ${ }^{26}$. The general idea behind it is quite natural: time series consist of repeated basis patterns of various sizes so the series can be decomposed on these patterns. Therefore, $L Z$ is the number of basis patterns. In this work, the original LZ76 was chosen for estimating the complexity. We further describe, in an easy way, how LZ76 algorithm works and how complexity can be calculated.

1. The original sequence $S=a_{1} a_{2} a_{3} \ldots a_{n}$, where $a_{i}$ is a number 0 or 1 , and $n$ is the number of elements in the time series, can be divided into subsequences:

$\mathrm{s}(\mathrm{i}, \mathrm{j})=\mathrm{a}_{\mathrm{i}} \mathrm{a}_{\mathrm{i}+1} \ldots \mathrm{a}_{\mathrm{j}}$, where $i \leq j$. The sequence $\mathrm{S}$ can then be written as the sum of subsequences: $S=s(1, i) s(i+1, j) \ldots s(k+1, n)$.

2. There are a lot of possibilities to divide the original time sequence $S$ in subsequences s. In LZ76 algorithm S is divided into subsequences that fulfill the relation: $s(i, j)$ is not a subsequence of $s(1, j-1)$. Only the last subsequence can be observed previously if there are not enough data.

3. The number of unique subsequences is defined as LZ complexity. For example, a binary sequence $\mathrm{S}=1001111011000010$ can be rewritten as: $1|0| 01|1110| 1100 \mid 0010$ which results in a complexity of $S$ equal to 6 . The binary sequence $S=111111$ has complexity 2 because, according to the second step in the LZ76 calculation, it can be rewritten as $1 \mid 11111$. 
The complexity depends on the time series length. To compare time series differing in length, it is necessary to define normalized complexity ${ }^{27,28}$. It has been shown that the upper bound of complexity $\mathrm{c}(\mathrm{N})$ is defined by:

$$
c(S)<\frac{N}{\left(1-\varepsilon_{\mathrm{N}}\right) \log _{\sigma}(N)}
$$

where $\mathrm{N}$ is the length of sequence and $\sigma$ is the number of different symbols in sequence ${ }^{19}$. For a binary sequence $\sigma=2(0$ and 1$), \varepsilon_{\mathrm{N}}$ is a slowly decaying function of $\mathrm{N}$, and for $N \rightarrow \infty, \varepsilon_{N}$ $\rightarrow 0$, therefore the normalized LZ76 complexity, C(S), can be calculated as ${ }^{19}$.

$$
C(S)=\frac{c(S) \log _{\sigma}(N)}{N}
$$

The impact of different lengths

It should be noted that the LZ76 complexity highly depends on the length of the strings. Although the first three phases of our experiment could be controlled in terms of length of time, due to the variability of the RR intervals lengths, two patients with the same length of the recordings did not have the same numbers of RR intervals. Therefore, the average $( \pm S D)$ number of RR intervals in our dataset was as follows: Phase 1 was $1284.13 \pm 149.23$, Phase 2 229.93 \pm 43.03 , Phase $31323.6 \pm 193.2$, and Phase 4 411.63 \pm 193.31 . This discrepancy of lengths might be a major drawback for the results, prone to biased conclusions, but could be minimized with normalization of LZ76 complexity.

To overcome the potential bias that results from the different lengths of the recordings, and not to discard any data, besides normalizing the LZ76 complexity, the shortest recording (94 RR intervals in one patient of Phase 4) was used as a reference length. Specifically, the shortest length was rounded to 90 datapoints. Instead of randomly selecting a length of 90 datapoints, which could further result in bias, the normalized LZ76 complexity 
was calculated in each recording using a box of length of 90 datapoints with 89 datapoints overlapping. Finally, after calculating the last box in a particular recording, the average of the obtained normalized LZ76 complexities were used as a measure of complexity for the recording.

\section{Statistical analysis}

The data were analyzed with Python 3 and the Matlab R2007a softwares. The normality of the distributions was assessed using the Kolmogorov-Smirnov test. The data are descriptively presented with means and standard deviations. Repeated measures ANOVA, was applied to assess the mean differences of the pNNx statistics and LZ76 complexity between phases. A ROC curve analysis was performed to test the model's diagnostic properties of pNNx and LZ76 as well as their combination, to differentiate stress phases (Phase 2 and 4) from the first baseline phase (Phase 1). $P$ values less than 0.05 were considered statistically significant.

Since $p N N x$ and $L Z 76$ were calculated for $x=1 \ldots 100$, the ROC calculation resulted in a series of 100 elements. Based on this ROC series ROCArea, we derived new features from pNNx and LZ76 values as follows:

$$
\begin{aligned}
& \overline{p N N x_{a-b}}=\frac{1}{b-a+1} \sum_{i=a}^{b} p N N x_{i} \\
& \overline{L Z 76_{a-b}}=\frac{1}{b-a+1} \sum_{i=a}^{b} L Z 76_{i}
\end{aligned}
$$

where, $a$ and $b$ are the starting and ending index of a sequence that meets the criteria $\forall i:$ ROCArea $_{i} \geq 0.7 \mid a \leq i \leq b$ and $b-a+1 \geq 5$. The descriptive statistics (mean and standard deviations), $p$ value and ROC area of these features were then calculated in the same way as mentioned before in this section. In addition, performance (ROC area) of different 
combinations of these features namely, I) all pNNx derived features; II) all LZ76 derived features; and III) both pNNx and LZ76 band features, were then computed using linear regression model with an intercept, linear terms, interactions, and squared terms. 


\section{RESULTS}

The results of the pNNx and LZ76 analysis are presented in the tables and further reported in figures. Due to the wide range, the results for the first ten NNx values are reported, while the following NNx values are reported rounded to the nearest tenth, i.e. $20,30,40$ etc. Table 1. contains mean values and standard deviation of the varying pNNx results across the different phases of the experiment. A common feature of all phases is the high percentage of pNNx values at lower NNx values, which strongly decreases when increasing the NNx values (Figure 1).

However, the stress phases can be visually differentiated from resting phases. An overlap between the resting phases, and both stress phases is observed. The difference of the curves is the lowest at the beginning and at the end of the $\mathrm{NNx}$ spectrum, and the widest at levels between NN10 and NN20.

The highest pNNx values are trivially observed for each phase at NN1. pNN1 values for the resting phases (phase $1-84.15 \pm 10.02$, phase $3-81.73 \pm 12.48$ ) were significantly higher than at the resting phases (phase $2-73.63 \pm 12.53$, phase $4-69.13 \pm 15.51$ ), $p<0.001$.

The value of the standard pNN50 at phase 1 and phase 3 were close to each other (4.53 \pm 6.46 , and $4.93 \pm 8.8$, respectively), similar to the values of pNN50 of phase 1 and phase $3(2.18 \pm 5.092 .38 \pm 4.23$, respectively), $p=0.021$

Considering statistical significance, a slow increase of $p$-values is observed with increasing NNx levels. The lowest p-values are seen at the first twenty NNx levels, while a cutoff is observed around NN70 (0.048), showing no statistically significant differences of pNNx levels above. 
The shape of the normalized LZ76 values for different pNNx values represents a right tailed function, with the peak values at lower pNNx values, and lags between phases (Figure 2). The highest LZ76 values for the resting phases are observed at NN5 and NN6 (phase 1: NN5 $-0.98 \pm 0.16$, NN6 $-0.98 \pm 0.17$, phase 3 : NN5 $-0.93 \pm 0.19$, NN6 $-0.93 \pm 0.21)$, and for the stress phases at NN3 (phase $2-0.96 \pm 0.17$, phase $4-0.93 \pm 0.18$ ). At NN50 LZ76 were significantly lower (phase $1-0.32 \pm 0.2$, phase $3-0.31 \pm 0.24$ ), with both resting phases having similar value, as well as the stress phases (phase $2-0.24 \pm 0.18$, phase $4-0.23 \pm 0.14$ ). Nonsignificant $p$-values were observed at NN3, NN4, and at NN leves greater than 80 (Table 2.).

The results of the ROC analysis are presented in Figures 3 and 4. A bimodal distribution is observed in the value of AUC levels with varying $N N$ increments for the pNNx statistics. Regarding the properties differenciating Phase 1 from Phase 2, the highest AUC were observed at lower NN increments, followed by a fall with AUC levels bellow 0.68 , NN30 having the smallest AUC value. An upward trend is further observed, with AUC levels plateauing between NN50 and NN75, followed again by a decrease in AUC levels.

A similar pattern is observed differenciating Phase 1 and Phase 4, however, the bimodal distribution flattened. The highest AUC values were observed at the smallest NN increments, followed by a slight decrease and then by an upward trend between NN 40 and 60. In general, except for the lowest NN increment, the AUC values were lower when discriminating Phase 1 from Phase 4, than Phase 1 from Phase 2.

The AUC values of the LZ76 complexity are presented in Figure 4. A similar pattern of the AUC values is observed when discriminating Phase 1 from Phase 2, and Phase 1 from Phase 4. A sudden decrease of AUC values is observed in both cases at lower NN increments. The highest AUC values differentiating Phase 1 from Phase 2 are observed at values around 60, 
while the highest values differentiating Phase 1 from Phase 4 are observed with NN increments between 10 and 20 .

Performances of derived features and their different combinations are presented in Table 3 and 4. Mean value of individual feature is higher in baseline phase (Phase 1) than stressed phase (Phase 2 and 4). The $p$ value clearly indicates that values of all features are statistical significantly different $(p<0.05)$ between baseline and stressed phases. The ROC area $(\geq 0.71$ ) also supports the findings. Table 4 shows the performance of different combinations of derived features. It is obvious that combining features together improves the differentiation capacity of derived features. The maximum ROC area between Phase 1 and 2 is 0.88 (C.I.: $0.80-0.94$ ) using all four derived features compared to 0.75 for the single best feature $\left(\overline{p N N x_{1-16}}\right)$. Similarly, combinations of all derived features shows higher ROC area 0.85 (C.I.: $0.75-0.91$ ) than 0.76 obtained using the best single feature $\left(\overline{p N N x_{1-15}}\right)$ to differentiate Phase 1 and 4. 


\section{DISCUSSION}

The first study stating the limitations of a single threshold value of $50 \mathrm{~ms}$ was conducted by Mietus et al ${ }^{16}$. Their findings showed that the selection of a wide range of thresholds would be more beneficial instead on relying on a single member of the pNNx family.

When comparing the graphical presentations of the $\mathrm{pNNx}$ values between healthy subjects and those suffering from congestive heart failure (CHF), a downward shift of the curves of CHF patients was observed ${ }^{16}$. This finding can be related to the results obtained in our study (Figure 1). The slope of the mean pNNx curves varies, with the stress phases decreasing pNNx much faster than resting phases, resulting in a downward shift of the stress phases curves.

Mietus et al. also stated that $\mathrm{NNx}<8$ might be likely of limited reliability in ECG data obtained with lower sampling rates, and potentially useful at higher sampling rates ${ }^{16}$. The monitor used in this study had a sampling rate of $1024 \mathrm{~Hz}$, and the obtained results show that very low thresholds can be applied using a high-resolution monitor, finding subtle variations in the heart rate

Generalizing the pNNx family opens further possibilities of more complex analysis. Yi et al. used a symbolic dynamics approach to the analysis of the pNNx statistics comparing healthy subjects, with patients suffering from atrial fibrillation (AF) and CHF patient group ${ }^{29}$. The mean pNNx curves were similar to the results reported by Mietus et al., while the curve of patients suffering from atrial fibrillation showed a slow negative downward trend, lacking the decrease ${ }^{16}$. In combination with a symbolic dynamics approach, the authors concluded 
that the optimal threshold for distinguishing healthy from sick patients would be between 10 and 25 , being far below from $50 \mathrm{~ms}^{29}$.

In our study, we considered the application of LZ complexity analysis as an alternative approach to the symbolic dynamics of a coarse-grained time series based on the binarized pNNx sequence.

The conventional 50 ms threshold showed statistically significant differences between resting and stressed newborns. For every threshold level, the complexity was greater in the resting phases, except for $\mathrm{NN}>1$ to $\mathrm{NN}>4$. However, no statistical significance differences were observed for NN3 and NN4.

The interpretation of the LZ complexity results can be explained in terms of a random sequence or the quantity of frequency components. The complexity of a completely random sequence tends to be 1 , while a periodic sequence has a complexity 0 . The greater the complexity of a sequence is, the more irregular and random the sequence is, and in contrary, the more periodic or the less regular the sequence is, the lesser is its complexity ${ }^{30}$. The results of the LZ76 complexity can be related to the pNNx statistics. A high threshold contains only a small fraction of the beats greater than the defined threshold itself, while a low threshold contains a majority of the values. For example, the probability of NN>1 in phase 1 is $84.15 \%$, while the probability of $N N>100$ is only $0.64 \%$. That means, there is a higher probability to obtain a uniform binarized sequence with pNN100, than pNN1. Those low frequency components result in a very low complexity of the higher threshold series.

The physiological interpretation of this behavior is not completely understood, and can currently only be speculated. HRV analysis has been already applied in the neonatal framework, using a multi-lag tone entropy, asymmetric detrended fluctuation, asymmetric Poincaré plot approach, and estimating the Hurst exponent using a mean-reversion 
approach $^{22-25}$. The increase of the negative scaling exponent, depicting higher autocorrelation, and the Poincaré plot asymmetry showed a higher contribution of accelerations within the RR interval time series, while the multi-lag tone-entropy showed a decrease of the parasympathetic, and an increase of the sympathetic branch of the autonomic nervous system 22-24. The reduction of pNNx values, and a consequential downward shift of the curve probably resulted as a modulation of the autonomic nervous system in stressed newborns, due to the reduction of the tone of the parasympathetic and increase of the sympathetic tone. The different complexities within resting and stressed newborns are a result of both a modulation of the autonomic nervous system and coarse-grained pNNx time series. Unfortunately, although the differences in the complexities were found between resting and stressed newborns, an explicit physiological interpretation cannot be stated yet. The reason for this is the wide range of $\mathrm{NN}$ thresholds, but in general, for threshold levels higher than $4 \mathrm{~ms}$, stress results in a decreased complexity. This finding is consistent with the reduction of entropy, reflecting the reduction of the parasympathetic and increasing the sympathetic activity ${ }^{23}$.

Another aim of this study is to utilize the newly observed feature extraction as a diagnostic tool, differentiating stressed from resting newborns. Questions may arise: how would it be beneficial using a complex method such as the LZ76 complexity, instead of an easyto-calculate, proven method like pNNx? The results of the ROC analysis show that the highest pNNx value for discriminating stressed from resting infants are at lower threshold levels. The AUC levels for LZ76 were even lower. However, when combined, different features from the pNNx family and LZ76 complexity resulted in an AUC of 0.88 with an upper bound of the confidence interval up to 0.94 for discriminating phase 2 from phase 1 , and AUC of 0.85 with a confidence interval up to 0.91 , for discriminating phase 4 from phase 1 . Although the confidence intervals showed no statistically significant results between the discrimination of 
different stress phases, it should be noted that different features were extracted to obtain a useful model. The reason for that difference is the duration and type of the applied stress. In phase 2, the heel was bluntly stimulated, while in phase 4, a sharp heel lance was applied, followed with intermittent heel pressing, to obtain the lowest amount of blood sufficient for metabolic screening. Nevertheless, the findings suggest that a combination of pNNx and LZ76 provides better results in discriminating stressed from resting newborns than each statistic alone.

Although this study is a result of a controlled acute stress, the findings show a promising application in real life. If a newborn is suspected to have a pathological condition, it will definitely experience procedural pain, and with a worsening condition, the newborn will be monitored in intensive care units using a heart rate monitor. One of the most illustrative examples using pNNx and LZ76, along with other HRV features would be analgesia and sedation titration. Both have various side effects which require caution when being applied, therefore it is crucial to have the child monitored, without unwanted exaggeration. Besides having a potential application in the pediatric field, the binarization of the pNNx time series opens possibilities for applying different nonlinear and complexity measures, i.e. LZ78, symbolic dynamics, entropy measures etc.

In conclusion, a novel HRV model was applied on neonates undergoing acute stress. This study stresses the importance of not solely relying on a single fixed threshold, but on a wide range of numbers. The traditional pNNx statistics can be further extended applying LZ76 complexity, which when combined makes it useful as an extension of the traditional methods for assessing stress and pain in this particularly vulnerable population. 


\section{Acknowledgements}

We thank $d r$. Marko Pirić, for their help in conducting the research.

\section{Conflict of interest}

None to declare. 


\section{REFERENCES}

1. Gunnar MR, Hertsgaard L, Larson M, Rigatuso J. Cortisol and behavioral responses to repeated stressors in the human newborn. Dev. Psychobiol. 24:487-505, 1991.

2. Kramarić K, Šapina M, Milas V, et al. The effect of ambient noise in the NICU on cerebral oxygenation in preterm neonates on high flow oxygen therapy. Signa Vitae. 13:52-56, 2017.

3. Anand KJ, Hickey PR. Pain and its effects in the human neonate and fetus. N. Engl. J. Med. 317:1321-1329, 1987.

4. Bauchner H, May A, Coates E. Use of analgesic agents for invasive medical procedures in pediatric and neonatal intensive care units. J. Pediatr. 121:647-649, 1992.

5. Grunau RE, Holsti L, Haley DW, et al. Neonatal procedural pain exposure predicts lower cortisol and behavioral reactivity in preterm infants in the NICU. Pain. 113:293-300, 2005.

6. Hatfield LA. Neonatal pain: What's age got to do with it? Surg. Neurol. Int. 5:S479, 2014.

7. Vesoulis ZA, Mathur AM. Cerebral autoregulation, brain injury, and the transitioning premature infant. Front. Pediatr. 5:64, 2017.

8. Stone LS, Szyf M. The emerging field of pain epigenetics. Pain. 154:1-2, 2013.

9. Blauer T, Gerstmann D. A simultaneous comparison of three neonatal pain scales during common NICU procedures. Clin. J. Pain. 14:39-47, 1998.

10. Boyle EM, Freer Y, Wong CM, Mclntosh N, Anand K. Assessment of persistent pain or distress and adequacy of analgesia in preterm ventilated infants. Pain. 124:87-91, 2006.

11. Storm H. The capability of skin conductance to monitor pain compared to other physiological pain assessment tools in children and neonates. Pediatric and Therapeutics. 3:2013. 
12. Faye PM, De Jonckheere J, Logier $\mathrm{R}$, et al. Newborn infant pain assessment using heart rate variability analysis. Clin. J. Pain. 26:777-782, 2010.

13. Pongiglione G, Fish FA, Strasburger JF, Benson DW. Heart rate and blood pressure response to upright tilt in young patients with unexplained syncope. J. Am. Coll. Cardiol. 16:165-170, 1990.

14. Cardiology TFotESo. Heart rate variability, standards of measurement, physiological interpretation, and clinical use. Circulation. 93:1043-1065, 1996.

15. Ewing DJ, Neilson J, Travis P. New method for assessing cardiac parasympathetic activity using 24 hour electrocardiograms. Heart. 52:396-402, 1984.

16. Mietus J, Peng C, Henry I, Goldsmith R, Goldberger A. The pNNx files: re-examining a widely used heart rate variability measure. Heart. 88:378-380, 2002.

17. Voss A, Schulz S, Schroeder R, Baumert M, Caminal P. Methods derived from nonlinear dynamics for analysing heart rate variability. Philos. Trans. Royal Soc. A. 367:277-296, 2008.

18. Sassi R, Cerutti S, Lombardi F, et al. Advances in heart rate variability signal analysis: joint position statement by the e-Cardiology ESC Working Group and the European Heart Rhythm Association co-endorsed by the Asia Pacific Heart Rhythm Society. Ep Europace. 17:1341-1353, 2015.

19. Lempel A, Ziv J. On the complexity of finite sequences. IEEE Trans. Inf. Theory. 22:7581, 1976.

20. Ferrario $M$, Signorini MG, Magenes $G$. Complexity analysis of the fetal heart rate variability: early identification of severe intrauterine growth-restricted fetuses. Med. Biol. Eng. Comput. 47:911-919, 2009.

21. Fernández A, López-Ibor M-I, Turrero A, et al. Lempel-Ziv complexity in schizophrenia: a MEG study. Clin. Neurophysiol. 122:2227-2235, 2011. 
22. Kramarić $K$, Šapina $M$, Garcin $M$, et al. Heart rate asymmetry as a new marker for neonatal stress. Biomed. Signal Process Control. 47:219-223, 2019.

23. Šapina M, Karmakar CK, Kramarić K, et al. Multi-lag tone-entropy in neonatal stress. J. Royal Soc. Interface. 15:20180420, 2018.

24. Šapina $M$, Kośmider $M$, Kramarić $K$, et al. Asymmetric detrended fluctuation analysis in neonatal stress. Physiol. Meas. 39:085006, 2018.

25. Šapina $M$, Garcin $M$, Kramaric K, Milas K, Brdaric D, Piric M. The Hurst exponent of heart rate variability in neonatal stress, based on a mean-reverting fractional Lévy stable motion. 2017.

26. Aboy M, Hornero R, Abásolo D, Álvarez D. Interpretation of the Lempel-Ziv complexity measure in the context of biomedical signal analysis. IEEE Trans. Biomed. Eng. 53:2282-2288, 2006.

27. Ziv J, Lempel A. Compression of individual sequences via variable-rate coding. IEEE Trans. Inf. Theory. 24:530-536, 1978.

28. Rapp P, Cellucci C, Korslund K, Watanabe T, Jimenez-Montano M. Effective normalization of complexity measurements for epoch length and sampling frequency. Phys Rev. E. 64:016209, 2001.

29. Yi S-H, Park K-T, Yoo C-S, Jeon G-R. Implementation of the Real-time Algorithms Based on the Symbolic Dynamics of a Coarse-grained Heart Rate Variability in Ubiquitous Health Care Systems. Paper presented at: World Congress on Medical Physics and Biomedical Engineering 20062007.

30. Wang J, Cui L, Wang H, Chen P. Improved complexity based on time-frequency analysis in bearing quantitative diagnosis. Adv. Mech. Eng. 5:258506, 2013. 


\section{TABLES}

Table 1. Mean \pm SD (standard deviation) of pNNx values (in percentage) across the different phases of the protocol with varying $\mathrm{pNNx}$

\begin{tabular}{|c|c|c|c|c|c|}
\hline & Phase 1 & Phase 2 & Phase 3 & Phase 4 & $\mathrm{p}^{*}$ \\
\hline pNN1 & $84.15 \pm 10.02$ & $73.63 \pm 12.53$ & $81.73 \pm 12.48$ & $69.13 \pm 15.51$ & $<0.001$ \\
\hline pNN2 & $75.63 \pm 13.1$ & $61.27 \pm 15.4$ & $72.7 \pm 16.36$ & $58.35 \pm 16.47$ & $<0.001$ \\
\hline pNN3 & $67.96 \pm 15.01$ & $51.6 \pm 16.55$ & $65.04 \pm 18.44$ & $49.33 \pm 16.74$ & $<0.001$ \\
\hline pNN4 & $61.16 \pm 16.32$ & $44.12 \pm 17.23$ & $58.49 \pm 19.43$ & $42.5 \pm 16.59$ & $<0.001$ \\
\hline pNN5 & $55.76 \pm 17.16$ & $38.77 \pm 17.52$ & $53.25 \pm 19.96$ & $37.38 \pm 16.56$ & $<0.001$ \\
\hline pNN6 & $50.69 \pm 17.69$ & $34.13 \pm 17.19$ & $48.62 \pm 20.26$ & $32.66 \pm 16.34$ & $<0.001$ \\
\hline pNN7 & $46.13 \pm 17.86$ & $30.03 \pm 16.49$ & $44.43 \pm 20.4$ & $29.31 \pm 15.62$ & $<0.001$ \\
\hline pNN8 & $42.21 \pm 18.04$ & $26.53 \pm 15.63$ & $40.5 \pm 20.37$ & $26.04 \pm 14.94$ & $<0.001$ \\
\hline pNN9 & $38.95 \pm 17.97$ & $23.85 \pm 15.06$ & $37.4 \pm 20.15$ & $23.85 \pm 14.36$ & $<0.001$ \\
\hline pNN10 & $35.76 \pm 17.81$ & $21.53 \pm 14.43$ & $34.53 \pm 19.91$ & $21.65 \pm 13.74$ & $<0.001$ \\
\hline pNN20 & $17.29 \pm 14.76$ & $9.92 \pm 10.63$ & $17.2 \pm 16.93$ & $9.92 \pm 10.04$ & $<0.001$ \\
\hline pNN30 & $10.15 \pm 11.48$ & $5.5 \pm 8.24$ & $10.52 \pm 13.95$ & $5.53 \pm 7.54$ & 0.005 \\
\hline pNN40 & $6.8 \pm 8.67$ & $3.37 \pm 6.65$ & $7.1 \pm 11.35$ & $3.62 \pm 6.09$ & 0.012 \\
\hline pNN50 & $4.53 \pm 6.46$ & $2.18 \pm 5.09$ & $4.93 \pm 8.8$ & $2.38 \pm 4.23$ & 0.021 \\
\hline pNN60 & $3.02 \pm 4.98$ & $1.5 \pm 4.03$ & $3.51 \pm 6.68$ & $1.55 \pm 2.93$ & 0.031 \\
\hline pNN70 & $2.12 \pm 3.91$ & $1.15 \pm 3.26$ & $2.63 \pm 5.22$ & $1.09 \pm 2.11$ & 0.048 \\
\hline pNN80 & $1.56 \pm 3.05$ & $0.84 \pm 2.53$ & $1.94 \pm 4.12$ & $0.84 \pm 1.77$ & 0.089 \\
\hline pNN90 & $1.07 \pm 2.21$ & $0.54 \pm 1.72$ & $1.36 \pm 2.99$ & $0.65 \pm 1.41$ & 0.119 \\
\hline pNN100 & $0.64 \pm 1.48$ & $0.38 \pm 1.25$ & $0.91 \pm 2.03$ & $0.44 \pm 0.93$ & 0.167 \\
\hline
\end{tabular}

*Repeated measures ANOVA 
Table 2. Mean \pm SD (standard deviation) of LZ76 values across the different phases of the protocol with varying $\mathrm{pNNx}$

\begin{tabular}{|c|c|c|c|c|c|}
\hline & Phase 1 & Phase 2 & Phase 3 & Phase 4 & $p^{*}$ \\
\hline NN1 & $0.67 \pm 0.16$ & $0.82 \pm 0.21$ & $0.7 \pm 0.2$ & $0.79 \pm 0.18$ & $<0.001$ \\
\hline NN2 & $0.82 \pm 0.17$ & $0.93 \pm 0.17$ & $0.82 \pm 0.18$ & $0.9 \pm 0.19$ & 0.001 \\
\hline NN3 & $0.91 \pm 0.15$ & $0.96 \pm 0.17$ & $0.89 \pm 0.17$ & $0.93 \pm 0.18$ & 0.121 \\
\hline NN4 & $0.96 \pm 0.16$ & $0.94 \pm 0.18$ & $0.92 \pm 0.18$ & $0.9 \pm 0.18$ & 0.382 \\
\hline NN5 & $0.98 \pm 0.16$ & $0.91 \pm 0.2$ & $0.93 \pm 0.19$ & $0.87 \pm 0.2$ & 0.013 \\
\hline NN6 & $0.98 \pm 0.17$ & $0.88 \pm 0.23$ & $0.93 \pm 0.21$ & $0.83 \pm 0.23$ & $<0.001$ \\
\hline NN7 & $0.97 \pm 0.18$ & $0.83 \pm 0.24$ & $0.92 \pm 0.22$ & $0.8 \pm 0.24$ & $<0.001$ \\
\hline NN8 & $0.95 \pm 0.18$ & $0.8 \pm 0.25$ & $0.9 \pm 0.23$ & $0.75 \pm 0.24$ & $<0.001$ \\
\hline NN9 & $0.93 \pm 0.19$ & $0.77 \pm 0.26$ & $0.88 \pm 0.23$ & $0.73 \pm 0.25$ & $<0.001$ \\
\hline NN10 & $0.9 \pm 0.2$ & $0.73 \pm 0.26$ & $0.86 \pm 0.24$ & $0.7 \pm 0.25$ & $<0.001$ \\
\hline NN20 & $0.63 \pm 0.25$ & $0.49 \pm 0.24$ & $0.61 \pm 0.27$ & $0.46 \pm 0.22$ & $<0.001$ \\
\hline NN30 & $0.46 \pm 0.25$ & $0.35 \pm 0.22$ & $0.45 \pm 0.27$ & $0.34 \pm 0.19$ & 0.002 \\
\hline NN40 & $0.37 \pm 0.22$ & $0.27 \pm 0.2$ & $0.37 \pm 0.26$ & $0.27 \pm 0.16$ & 0.003 \\
\hline NN50 & $0.32 \pm 0.2$ & $0.24 \pm 0.18$ & $0.31 \pm 0.24$ & $0.23 \pm 0.14$ & 0.012 \\
\hline NN60 & $0.27 \pm 0.17$ & $0.21 \pm 0.17$ & $0.28 \pm 0.22$ & $0.2 \pm 0.1$ & 0.016 \\
\hline NN70 & $0.24 \pm 0.15$ & $0.2 \pm 0.16$ & $0.25 \pm 0.19$ & $0.19 \pm 0.08$ & 0.044 \\
\hline NN80 & $0.22 \pm 0.13$ & $0.19 \pm 0.14$ & $0.23 \pm 0.17$ & $0.18 \pm 0.07$ & 0.057 \\
\hline NN90 & $0.2 \pm 0.1$ & $0.18 \pm 0.1$ & $0.21 \pm 0.13$ & $0.17 \pm 0.06$ & 0.108 \\
\hline NN100 & $0.18 \pm 0.08$ & $0.17 \pm 0.09$ & $0.19 \pm 0.1$ & $0.17 \pm 0.05$ & 0.231 \\
\hline
\end{tabular}

*Repeated measures ANOVA 
Table 3. Mean \pm SD (Standard Deviation) values of band features calculated from pNNx and LZ16 profiles. P value calculated using non-parametric Mann-Whitney $U$ test. ROC Area shows the phase differentiating (Phase 1 vs Phase 2 or Phase 1 vs Phase 4) capability of individual feature.

\begin{tabular}{|c|c|c|c|c|}
\hline FeatureName & $\begin{array}{l}\text { Phase } 1 \\
\text { (mean } \pm \text { SD) }\end{array}$ & $\begin{array}{l}\text { Phase } 2 \\
\text { (mean } \pm \text { SD) }\end{array}$ & $P$ & ROC Area \\
\hline$\overline{\text { Area }_{1-16}^{P N N X}}$ & $45.17 \pm 16.00$ & $31.37 \pm 13.92$ & $<0.001$ & 0.75 \\
\hline$\overline{\operatorname{Area}_{46-93}^{P N N X}}$ & $2.49 \pm 4.13$ & $1.27 \pm 3.39$ & $<0.001$ & 0.73 \\
\hline$\overline{\text { Area }_{39-43}^{L Z 76}}$ & $0.37 \pm 0.22$ & $0.27 \pm 0.20$ & 0.002 & 0.71 \\
\hline \multirow[t]{2}{*}{ Area $a_{46-98}^{L Z 76}$} & $0.25 \pm 0.14$ & $0.20 \pm 0.14$ & $<0.001$ & 0.74 \\
\hline & $\begin{array}{l}\text { Phase } 1 \\
\text { (mean } \pm \text { SD) }\end{array}$ & $\begin{array}{l}\text { Phase } 4 \\
\text { (mean } \pm \text { SD) }\end{array}$ & $P$ & ROC Area \\
\hline$\overline{\overline{A r e a}}{ }_{1-15}^{P N N X}$ & $46.68 \pm 16.04$ & $31.60 \pm 13.86$ & $<0.001$ & 0.76 \\
\hline$\overline{\text { Area }_{6-20}^{L Z 76}}$ & $0.81 \pm 0.19$ & $0.62 \pm 0.22$ & $<0.001$ & 0.75 \\
\hline$\overline{\text { Area }_{52-61}^{L Z 76}}$ & $0.29 \pm 0.18$ & $0.21 \pm 0.11$ & 0.001 & 0.72 \\
\hline$\overline{\text { Area }_{73-80}^{L Z 76}}$ & $0.23 \pm 0.14$ & $0.18 \pm 0.07$ & 0.001 & 0.71 \\
\hline
\end{tabular}

$\overline{\text { Area }_{1-16}^{P N N X}}$ represents the average area of $\mathrm{pNNx}$ feature profile, where $x \in[1,16]$. This range was calculated based on sequences of $x$ for which the ROC area is greater than 12 . 
Table 4. ROC Area and confidence interval obtained using pNNx, LZ16 and their combination in differentiating phases (Phase 1 vs Phase 2 and Phase 1 vs Phase 4). Multiple features were combined using a linear regression model.

\begin{tabular}{|c|c|c|}
\hline Phases & FeatureName & ROC Area (C.I.) \\
\hline \multirow[t]{3}{*}{ Phase 1 vs Phase 2} & $\overline{\text { Area }_{1-16}^{P N N X}}, \overline{\text { Area }}{ }_{46-93}^{P N N X}$ & $0.79(0.71-0.89)$ \\
\hline & $\overline{\text { Area }_{39-43}^{L Z 76}}$, Area $_{46-98}^{L Z 76}$ & $0.73(0.59-0.84)$ \\
\hline & $\overline{\operatorname{Area}_{1-16}^{P N N X}}, \overline{\text { Area }_{46-93}^{P N N X}}, \overline{\text { Area }_{39-43}^{L Z 76}}, \overline{\text { Area }_{46-98}^{L Z 76}}$ & $0.88(0.80-0.94)$ \\
\hline \multirow[t]{3}{*}{ Phase 1 vs Phase 4} & $\overline{\operatorname{Area}_{1-15}^{P N N X}}$ & $0.76(0.66-0.86)$ \\
\hline & $\overline{\operatorname{Area}_{6-20}^{L Z 76}}, \overline{\text { Area }_{52-61}^{L Z 76}}, \overline{\text { Area }_{73-80}^{L Z 76}}$ & $0.80(0.70-0.90)$ \\
\hline & $\overline{\operatorname{Area}_{1-15}^{P N N X}}, \overline{\text { Area }_{6-20}^{L Z 76}}, \overline{\text { Area }_{52-61}^{L Z 76}}, \overline{\text { Area }_{73-80}^{L Z 76}}$ & $0.85(0.74-0.91)$ \\
\hline
\end{tabular}

$\overline{\overline{A r e a}} a_{1-16}^{P N X}$ represents the average area of pNNx feature profile, where $x \in[1,16]$. This range was calculated based on sequences of $x$ for which the ROC area is greater than 12 . 


\section{FIGURES}

Figure 1: The distributions of mean $\mathrm{pNNx}$ values within each phase of the experiment for $\mathrm{NN}=[1,100]$

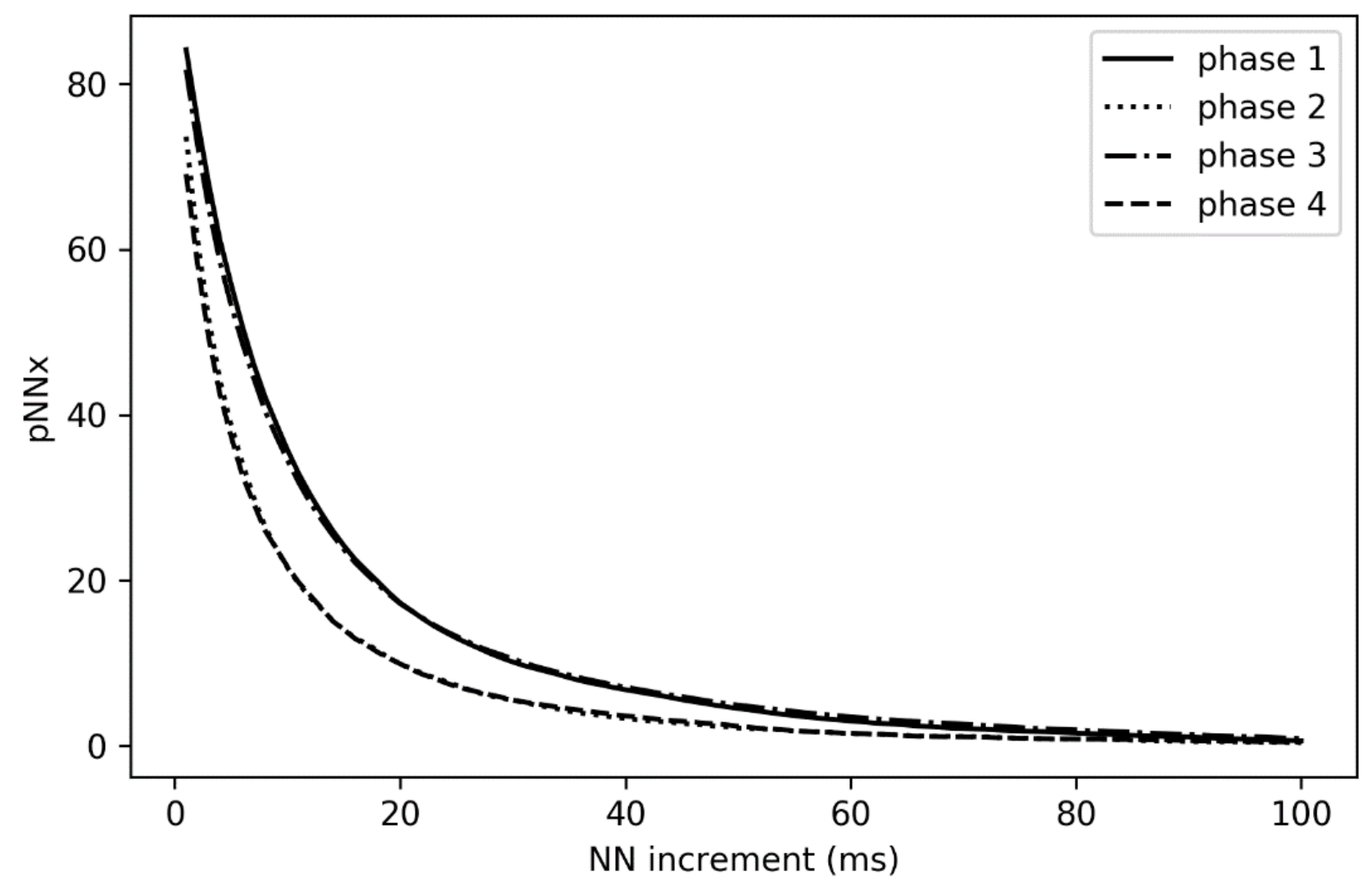

Phase 1 - first resting phase, Phase 2 - dummy stimulation phase, Phase 3 - second resting phase, Phase 4 - heel stick blood drawing phase 
Figure 2: The distributions of mean LZ76 within each phase of the experiment for $N N=[1,100]$

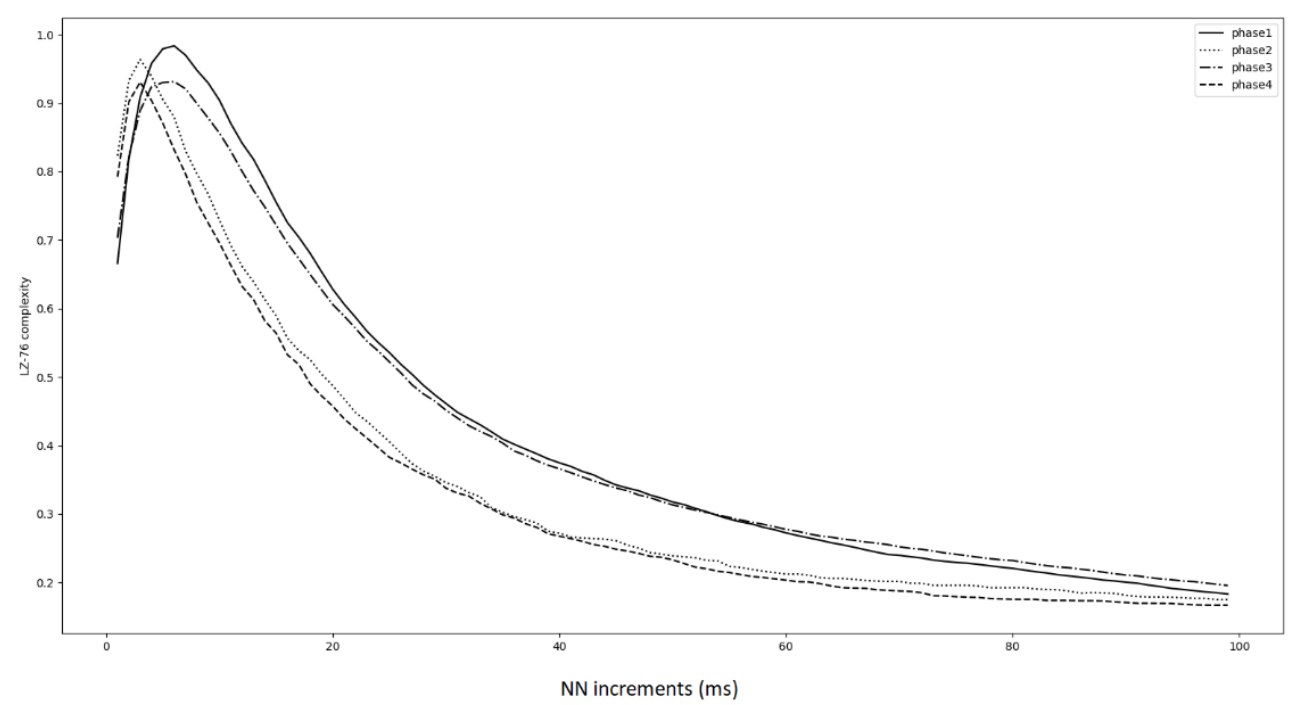

Phase 1 - first resting phase, Phase 2 - dummy stimulation phase, Phase 3 - second resting phase, Phase 4 - heel stick blood drawing phase 
Figure 3: ROC Area of pNNX feature for differentiating Phase 1 from Phase 2 and Phase1 from Phase 4 with varrying NN values.
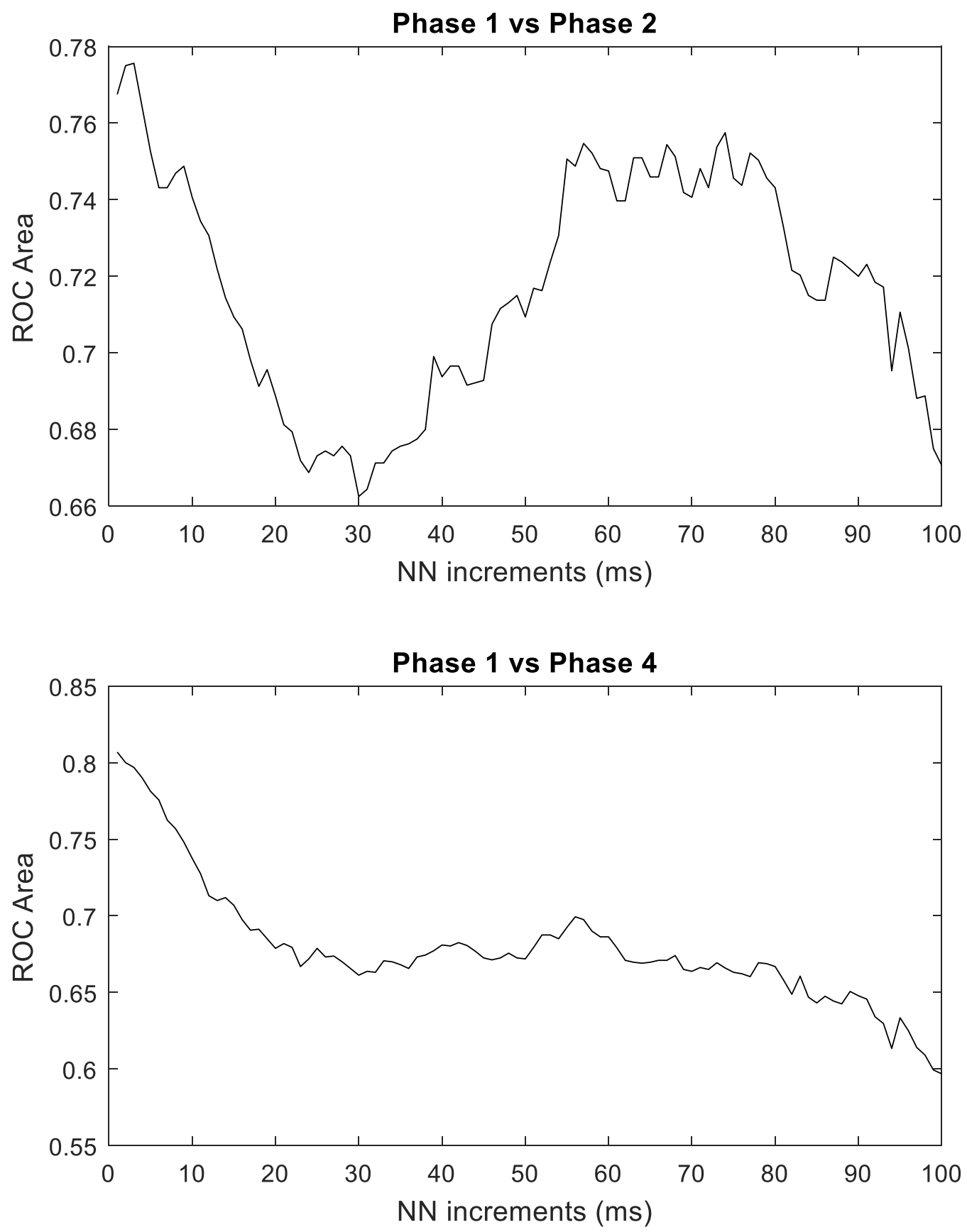
Figure 4: ROC Area of LZ76 feature for differentiating Phase 1 from Phase 2 and Phase 1 from Phase 4 with varrying NN values.
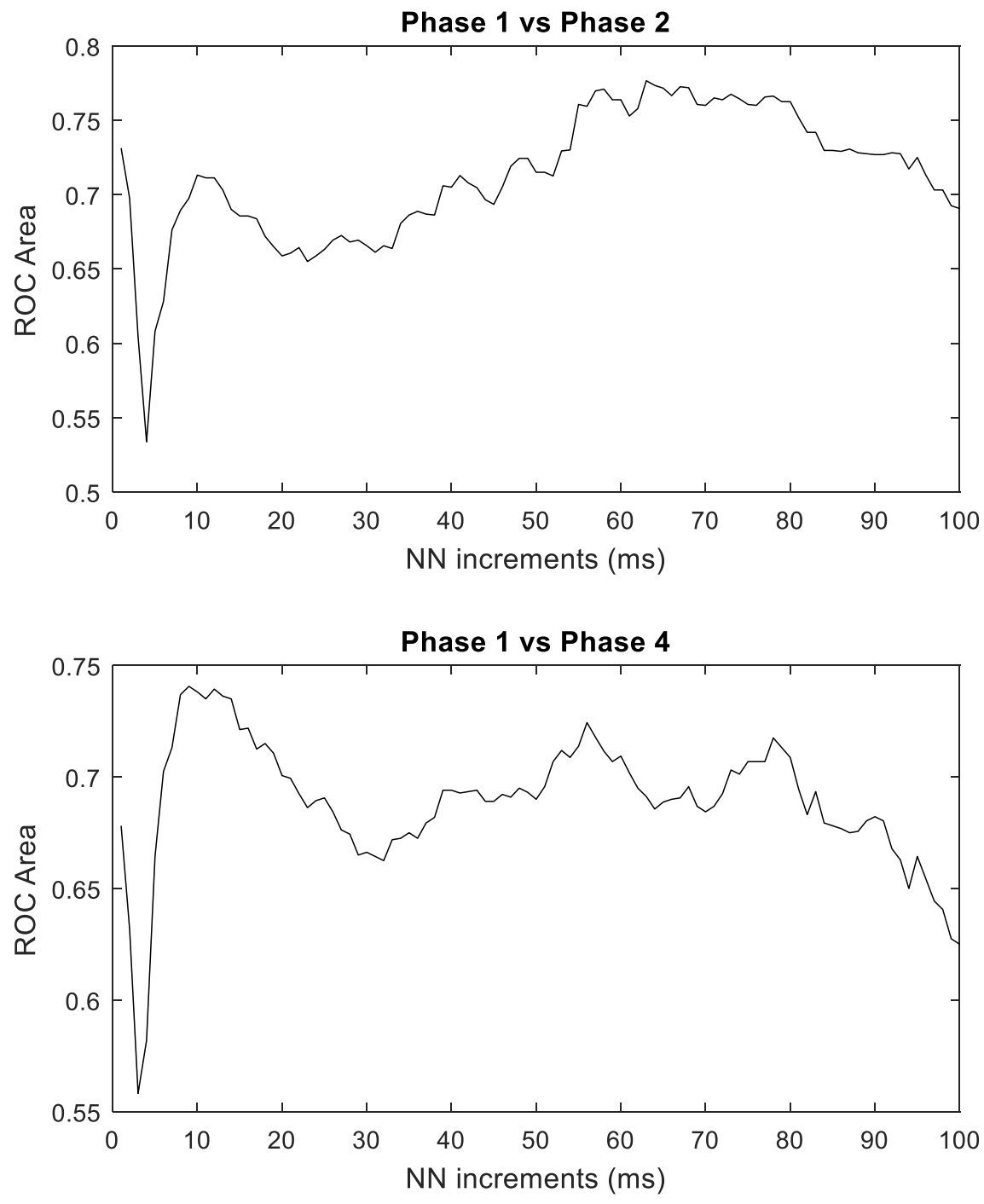